\title{
Power Loss for Inhomogeneous Poisson Processes
}

\author{
Fazli Kh. \\ Department of Mathematics, \\ University of Kurdistan, Sanandaj \\ khfazli@uok.ac.ir
}

November 9, 2018

\begin{abstract}
In this work, based on a realization of an inhomogeneous Poisson process whose intensity function depends on a real unknown parameter, we consider a simple hypothesis against a sequence of close (contiguous) alternatives. Under certain regularity conditions we obtain the power loss of the score test with respect to the Neyman-Pearson test. The power loss measures the performance of a second order efficient test by the help of third order asymptotic properties of the problem under consideration.
\end{abstract}

AMS 1991 Classification: 62M05.

Key words: Inhomogeneous Poisson processes, hypotheses testing, power loss, second order efficiency.

\section{Introduction}

Let $X^{(n)}$ be a realization of a nonhomogeneous Poisson process observed on some increasing subsets $\mathbb{A}_{n}, n=1,2, \ldots$ of $d$ dimensional Euclidian space $\mathbb{R}^{d}$ with intensity function $S(\vartheta, x), x \in \mathbb{A}_{n}$ depending on one-dimensional parameter $\vartheta \in \Theta$. Based on $X^{(n)}$ we want to test the hypotheses

$$
\begin{aligned}
& \mathcal{H}_{0}: \vartheta=\vartheta_{0} \\
& \mathcal{H}_{1}: \vartheta>\vartheta_{0},
\end{aligned}
$$


where $\vartheta_{0}$ is a given value in the parameter space $\Theta$. Let us fix some $\alpha \in(0,1)$ and define the class $\mathcal{K}_{\alpha}^{(n)}$ of tests at level $1-\alpha(\operatorname{size} \alpha)$, i.e.,

$$
\mathcal{K}_{\alpha}^{(n)}=\left\{\phi_{n}: \quad \mathbf{E}_{\vartheta_{0}} \phi_{n}\left(X^{(n)}\right)=\alpha\right\},
$$

where $\mathbf{E}_{\vartheta}$ denotes the mathematical expectation with respect to the probability measure $\mathbf{P}_{\vartheta}^{(n)}$. With fixed $n$, generally speaking, there is no uniformly most powerful test in $\mathcal{K}_{\alpha}^{(n)}$ (see [8]). Therefore we turn to the asymptotic approach and introduce the class $\mathcal{K}_{\alpha}^{\prime}$ of sequence of tests of asymptotic level $1-\alpha$, i.e.,

$$
\mathcal{K}_{\alpha}^{\prime}=\left\{\left\{\phi_{n}\right\}: \quad \lim _{n \rightarrow \infty} \mathbf{E}_{\vartheta_{0}} \phi_{n}\left(X^{(n)}\right)=\alpha\right\} .
$$

It is well known that if $n \rightarrow \infty$ for any given value $\vartheta$ of the alternative the power of any reasonable (consistent) test tends to 1 ( see [?], [1]). In order to compare the different tests we use the Pitman's approach (see [9], [7]) where instead of a fixed alternative $\vartheta$ we consider a sequence of socalled local alternatives (close or contiguous alternatives) which converges to $\vartheta_{0}$ with a certain rate and hence it is difficult to distinguish between the null hypothesis and alternative. More precisely, let $\left\{\varphi_{n}\right\}$ be a sequence of nonnegative numbers which converges to zero with such a rate that the likelihood ratio

$$
Z_{n}(u)=\frac{\mathrm{d} \mathbf{P}_{\vartheta_{0}+\varphi_{n} u}^{(n)}}{\mathrm{d} \mathbf{P}_{\vartheta_{0}}^{(n)}}\left(X^{(n)}\right)
$$

has a nondegenerate limit for any $u$ with $\vartheta_{0}+\varphi_{n} u \in \Theta$. By the NeymanPearson lemma the most powerful test for $\mathcal{H}_{0}: \vartheta=\vartheta_{0}$ against the local alternative $\mathcal{H}_{u}: \vartheta=\vartheta_{0}+\varphi_{n} u$ with $u>0$, is given by

$$
\tilde{\phi}_{n}\left(X^{(n)}\right)= \begin{cases}1, & \text { if } \Lambda_{n}(u)>b_{n}(u) \\ 0, & \text { if } \Lambda_{n}(u)<b_{n}(u)\end{cases}
$$

where $\Lambda_{n}(u)=\ln Z_{n}(u)$ and the constant $b_{n}(u)$ together with the contribution of the randomized part provide the size $\mathbf{E}_{\vartheta_{0}} \tilde{\phi}_{n}\left(X^{(n)}\right)=\alpha$. The power of $\tilde{\phi}_{n}$ as a function of $u$ is called the envelope power function. For any fixed $n$ it is the supremum of the power at the local alternative $\vartheta_{u}=\vartheta_{0}+\varphi_{n} u$ over all the tests at level $1-\alpha$, i.e.

$$
\mathbf{E}_{\vartheta_{u}} \tilde{\phi}_{n}\left(X^{(n)}\right)=\sup _{\phi_{n} \in \mathcal{K}_{\alpha}^{(n)}} \mathbf{E}_{\vartheta_{u}} \phi_{n}\left(X^{(n)}\right)
$$

Notice that $\tilde{\phi}_{n}$ is not a test for the main hypotheses $\mathcal{H}_{0}$ and $\mathcal{H}_{1}$ because it depends on the parameter $u$. 
Let us introduce the score statistic

$$
\Delta_{n}\left(\vartheta_{0}\right)=\varphi_{n} \int_{\mathbb{A}_{n}} \frac{\dot{S}\left(\vartheta_{0}, x\right)}{S\left(\vartheta_{0}, x\right)} \pi^{(n)}(\mathrm{d} x), \quad \varphi_{n}^{-2}=\int_{\mathbb{A}_{n}} \frac{\dot{S}\left(\vartheta_{0}, x\right)^{2}}{S\left(\vartheta_{0}, x\right)} \mathrm{d} x
$$

where the normalizing factor $\varphi_{n}=I_{n}\left(\vartheta_{0}\right)^{-1 / 2}$ is the inverse square root of the Fisher information

$$
I_{n}\left(\vartheta_{0}\right)=\int_{\mathbb{A}_{n}} \frac{\dot{S}\left(\vartheta_{0}, x\right)^{2}}{S\left(\vartheta_{0}, x\right)} \mathrm{d} x
$$

at the point $\vartheta_{0}$. Here $\pi^{(n)}(\mathrm{d} x)=X^{(n)}(\mathrm{d} x)-S\left(\vartheta_{0}, x\right) \mathrm{d} x$ is the centered Poisson process (for the definition of a stochastic integral w.r.t. a Poisson process see the next section) and $\dot{S}(\vartheta, x)$ denotes the derivative of $S(\vartheta, x)$ with respect to $\vartheta$. Based on $\Delta_{n}\left(\vartheta_{0}\right)$ we introduce the score test

$$
\bar{\phi}_{n}\left(X^{(n)}\right)= \begin{cases}1, & \text { if } \Delta_{n}\left(\vartheta_{0}\right)>z_{\alpha} \\ 0, & \text { if } \Delta_{n}\left(\vartheta_{0}\right) \leq z_{\alpha}\end{cases}
$$

where $z_{\alpha}$ is $1-\alpha$ quantile of standard Gaussian law, i.e., $\mathbf{P}\left\{\zeta>z_{\alpha}\right\}=\alpha$ and $\zeta \sim \mathcal{N}(0,1)$. It is well known that if the family $\left\{\mathbf{P}_{\vartheta}^{(n)}, \vartheta \in \Theta\right\}$ of distributions is locally asymptotically normal (LAN) at the point $\vartheta_{0}$, then the test $\bar{\phi}_{n} \in \mathcal{K}_{\alpha}^{\prime}$ is locally asymptotically uniformly most powerful (LAUMP) (or first order efficient), i.e., for any $K>0$

$$
\sup _{0 \leq u \leq K}\left|\mathbf{E}_{\vartheta_{u}} \tilde{\phi}_{n}\left(X^{(n)}\right)-\mathbf{E}_{\vartheta_{u}} \bar{\phi}_{n}\left(X^{(n)}\right)\right|=o(1)
$$

as $n \rightarrow \infty$ (see [12]). Moreover the power function of $\bar{\phi}_{n}$ at $\vartheta_{u}$ admits the representation

$$
\mathbf{E}_{\vartheta_{u}} \bar{\phi}_{n}\left(X^{(n)}\right)=\mathbf{P}\left\{\zeta>z_{\alpha}-u\right\}+o(1)
$$

for any $u>0$, where $\zeta \sim \mathcal{N}(0,1)$. For $n$ large, hence the power of $\bar{\phi}_{n}$ approximates the envelope power function up to order $o(1)$. A refinement of (11) is given in (4). For a family $\left\{\mathbf{P}_{\vartheta}^{(n)}, \vartheta \in \Theta\right\}$ of distributions related to a Poisson process with intensity functions $\{S(\vartheta, \cdot), \vartheta \in \Theta\}$ the conditions of LAN for multidimensional parameter $\vartheta$ are obtained by Yu. A. Kutoyants, [6]. The first order efficiency of $\bar{\phi}_{n}$ follows from the LAN representation which implies in turn the asymptotic normalities of $\Delta_{n}\left(\vartheta_{0}\right)$ and $\Lambda_{n}(u)$ under both $\mathcal{H}_{0}$ and $\mathcal{H}_{u}$. Therefore the refinement of the central limit theorem, by taking into account one term after the Gaussian term, improves the situation. 
This can be done by the help of Edgeworth type expansion of the distribution function of the stochastic integral $\Delta_{n}\left(\vartheta_{0}\right)$ and $\Lambda_{n}(u)$ under $\mathcal{H}_{0}$ and $\mathcal{H}_{u}$. Under certain regularity conditions related to second order asymptotic properties of the family $\left\{\mathbf{P}_{\vartheta}^{(n)}, \vartheta \in \Theta\right\}$, we can construct a second order efficient test, i.e., a test $\phi_{n}^{*}$ such that for any $K>0$

$$
\sup _{0 \leq u \leq K}\left|\mathbf{E}_{\vartheta} \tilde{\phi}_{n}\left(X^{(n)}\right)-\mathbf{E}_{\vartheta_{u}} \phi_{n}^{*}\left(X^{(n)}\right)\right|=O\left(\varepsilon_{n}^{2}\right),
$$

for some sequence $\varepsilon_{n} \rightarrow 0$ (see [4]). Furthermore the probability of the first type error of $\phi_{n}^{*}$ is given by $\mathbf{E}_{\vartheta_{0}} \phi_{n}^{*}\left(X^{(n)}\right)=\alpha+O\left(\varepsilon_{n}^{2}\right)$. From (2) it follows that the power function of $\phi_{n}^{*}$ (a second order efficient test, generally) approximates the envelope power function up to order $O\left(\varepsilon_{n}^{2}\right)$ and hence it works as good as $\tilde{\phi}_{n}$ up to this order. Second order efficiency of $\phi_{n}^{*}$ is related to the fact that the two first terms in the Edgeworth expansions of the distributions functions of $\Delta_{n}\left(\vartheta_{0}\right)$ and $\Lambda_{n}(u)$ under the local alternative are equal up to the order $O\left(\varepsilon_{n}^{2}\right)$. Hence to measure the performance of a second order efficient test, and especially $\phi_{n}^{*}$, it is natural to consider the power loss of $\phi_{n}^{*}$ with respect to the most powerful test $\tilde{\phi}_{n}$, which is defined by

$$
r(u)=\lim _{n \rightarrow \infty} \varepsilon_{n}^{-2}\left(\mathbf{E}_{\vartheta_{u}} \tilde{\phi}_{n}\left(X^{(n)}\right)-\mathbf{E}_{\vartheta_{u}} \phi_{n}^{*}\left(X^{(n)}\right)\right),
$$

for $u>0$. This requires to take into account higher order terms in the Edgeworth expansions of the distribution functions. See [1], chapter 3, for a general theorem and the power loss results for the tests based on $L-, R-$ and $U$ - statistics in the i.i.d. case. The main object of this work is to obtain the power loss of the score test $\phi_{n}^{*}$ based on a realization $X^{(n)}$ of a nonhomogeneous Poisson process with intensity function $S(\vartheta, x), x \in \mathbb{A}_{n}$ and to give the explicit representation of $r(u)$.

\section{Preliminaries}

Let us remind several facts from a Poisson process. A Poisson process $X^{(n)}$ is a random point measure which on the set $\mathbb{B} \subset \mathbb{A}_{n}$ can be written as

$$
X^{(n)}(\mathbb{B})=\sum_{x_{i} \in \mathbb{A}_{n}} \chi_{\left\{x_{i} \in \mathbb{B}\right\}},
$$

where $\left\{x_{i}\right\}$ are the events (random points) of the Poisson process and $\chi_{\{\mathbb{D}\}}$ is the indicator function of the event $\mathbb{D}$. The Poisson process with (parametric) intensity function $S(\vartheta, x), x \in \mathbb{A}_{n}$ (with respect to Lebesgue measure) is entirely defined by the following two conditions: 
- for any collection of disjoint sets $\mathbb{B}_{1}, \ldots, \mathbb{B}_{m} \subseteq \mathbb{A}_{n}$ the random variables $X^{(n)}\left(\mathbb{B}_{1}\right), \ldots, X^{(n)}\left(\mathbb{B}_{m}\right)$ are independent,

- the random variable $X^{(n)}(\mathbb{B})$ for any $\mathbb{B} \subseteq \mathbb{A}_{n}$ has Poisson distribution with parameter $\Lambda_{\vartheta}^{(n)}(\mathbb{B})=\int_{\mathbb{B}} S(\vartheta, x) \mathrm{d} x$.

This and other definitions of the spatial Poisson processes as well as their properties and examples can be found in many books devoted to point processes (see, e.g., Daley and Vere-Jones [2], Krickeberg [5], Reiss [10], Ripley [11], Snyder and Miller [13]). The spatial Poisson processes are widely used in many fields. In particular this is a good mathematical model for the problems of image restoration when the optical signal is weak and statistics of photons is well described by an inhomogeneous Poisson process [13].

By the definition, $X^{(n)}$ is a random element of the set $\mathcal{M}_{0}^{(n)}$, containing all integer valued measures defined on the set $\mathbb{A}_{n}$. Let $\mathfrak{B}\left(\mathcal{M}_{0}^{(n)}\right)$ be the smallest $\sigma$-field with respect to which all the mappings:

$$
\Pi_{\mathbb{B}}: \mathcal{M}_{0}^{(n)} \rightarrow\{0,1,2, \ldots, \infty\}, \quad \Pi_{\mathbb{B}}\left(X^{(n)}\right)=X^{(n)}(\mathbb{B}), \quad \mathbb{B} \in \mathcal{A}_{n},
$$

are measurable. Here $\mathcal{A}_{n}$ is the $\sigma$-field of Borel subsets of $\mathbb{A}_{n}$. Let $\mathbf{P}_{\vartheta}^{(n)}$ denote the probability law induced by the random element (realization) $X^{(n)}$ of a Poisson process with intensity function $S(\vartheta, x), x \in \mathbb{A}_{n}$ on the measurable space $\left(\mathcal{M}_{0}^{(n)}, \mathfrak{B}\left(\mathcal{M}_{0}^{(n)}\right)\right)$. We remind that if the intensity measures $\Lambda_{\vartheta_{1}}^{(n)}$ and $\Lambda_{\vartheta_{2}}^{(n)}$ are equivalent then the corresponding probability measures $\mathbf{P}_{\vartheta_{1}}^{(n)}$ and $\mathbf{P}_{\vartheta_{2}}^{(n)}$ are equivalent and the likelihood ratio is given by

$$
\begin{aligned}
& \frac{\mathrm{d} \mathbf{P}_{\vartheta_{2}}^{(n)}}{\mathrm{d} \mathbf{P}_{\vartheta_{1}}^{(n)}}\left(X^{(n)}\right)= \\
& =\exp \left\{\int_{\mathbb{A}_{n}} \ln \frac{S\left(\vartheta_{2}, x\right)}{S\left(\vartheta_{1}, x\right)} X^{(n)}(\mathrm{d} x)-\int_{\mathbb{A}_{n}}\left[S\left(\vartheta_{2}, x\right)-S\left(\vartheta_{1}, x\right)\right] \mathrm{d} x\right\} .
\end{aligned}
$$

For a proof see [6] page 28. Here the stochastic integral

$$
\int_{\mathbb{A}_{n}} f(x) X^{(n)}(\mathrm{d} x)=\sum_{x_{i} \in \mathbb{A}_{n}} f\left(x_{i}\right)
$$

where $\left\{x_{i}\right\}$ are the events (random points) of the Poisson process.

Since the main tool used in this work is based on the Edgeworth expansion, here we present the conditions under which the distribution function

$$
F_{n}(y)=\mathbf{P}_{\vartheta}^{(n)}\left\{I_{n}(f)<y\right\},
$$


of the stochastic integral

$$
I_{n}(f)=\int_{\mathbb{A}_{n}} f_{n}(x) \pi^{(n)}(\mathrm{d} x),
$$

admits an Edgeworth type expansion for two terms after the Gaussian term, where $\pi^{(n)}(\mathrm{d} x)=X^{(n)}(\mathrm{d} x)-S(\vartheta, x) \mathrm{d} x$ is the centered Poisson process. We suppose that

$$
\int_{\mathbb{A}_{n}} f_{n}(x)^{2} S(\vartheta, x) \mathrm{d} x=1
$$

The expansion is obtained under the following two conditions:

$\mathcal{B}_{1}$. There exists a sequence of real numbers $\varepsilon_{n} \rightarrow 0$, as $n \rightarrow \infty$ and constants $C_{r}>0, r=3,4,5$, such that

$$
\int_{\mathbb{A}_{n}}\left|f_{n}(x)\right|^{r} S(\vartheta, x) \mathrm{d} x \leq C_{r} \varepsilon_{n}^{r-2}
$$

$\mathcal{B}_{2}$. There exist constants $\gamma \geq 5 / 2$ and $c_{0}>0$ satisfying the inequality $\frac{C_{3}}{3 !} c_{0}+\frac{C_{4}}{4 !} c_{0}^{2}+\frac{C_{5}}{5 !} c_{0}^{3}-\frac{1}{2}<0$ such that

$$
\inf _{\frac{c_{0} \varepsilon_{n}^{-1}}{2}<t<\frac{\varepsilon_{n}^{-2}}{2}} \int_{\mathbb{A}_{n}} \sin ^{2}\left(t f_{n}(x)\right) S(\vartheta, x) \mathrm{d} x \geq \gamma \ln \varepsilon_{n}^{-1}
$$

for all large $n$.

Let us introduce the cumulants:

$$
\gamma_{r, n}=\int_{\mathbb{A}_{n}} f_{n}(x)^{r} S(\vartheta, x) \mathrm{d} x, \quad r=3,4
$$

and the Hermit polynomials:

$$
H_{2}(y)=y^{2}-1, \quad H_{3}(y)=y^{3}-3 y, \quad H_{5}(y)=y^{5}-10 y^{3}+15 y .
$$

Theorem 1. Let the conditions $\mathcal{B}_{1}, \mathcal{B}_{2}$ be fulfilled, then uniformly in $y \in \mathbb{R}$ $F_{n}(y)=\mathcal{N}(y)-\frac{\gamma_{3, n}}{3 !} H_{2}(y) n(y)-\frac{\gamma_{4, n}}{4 !} H_{3}(y) n(y)-\frac{\gamma_{3, n}^{2}}{72} H_{5}(y) n(y)+O\left(\varepsilon_{n}^{3}\right)$, for all $n$ large. Here $\mathcal{N}(y)$ and $n(y)$ denote the distribution and density functions of the standard Gaussian law, respectively. 
Note that $\gamma_{r, n}=O\left(\varepsilon_{n}^{r-2}\right), r=3,4$ by $\mathcal{B}_{1}$.

Proof. See [3], Page 36. The proof is a special case of a general theorem given by Kutoyants, where the expansion is obtained by the powers of $\varepsilon_{n}$ up to order $\varepsilon_{n}^{k}, k=1,2, \ldots$ (see ([6]), page 131).

Corollary 1. Let $0<\alpha<1$ be given and the conditions $\mathcal{B}_{1}-\mathcal{B}_{2}$ be fulfilled. Then the equation $F_{n}(y)=1-\alpha+O\left(\varepsilon_{n}^{3}\right)$ has a solution $y=c_{n, \alpha}$,

$$
c_{n, \alpha}=z_{\alpha}+\frac{\gamma_{3, n}}{6} H_{2}\left(z_{\alpha}\right)+\frac{\gamma_{4, n}}{24} H_{3}\left(z_{\alpha}\right)+\frac{\gamma_{3, n}^{2}}{72} H_{5}\left(z_{\alpha}\right) .
$$

See [3], page 40 for proof.

\section{Second Order Efficiency}

Let $\beta_{n}\left(u, \phi_{n}\right)$ denote the power of a test $\phi_{n}$ at the local alternative $\vartheta_{u}=$ $\vartheta_{0}+\varphi_{n} u$, i.e.,

$$
\beta_{n}\left(u, \phi_{n}\right)=\mathbf{E}_{\vartheta_{u}} \phi_{n}\left(X^{(n)}\right) .
$$

Under certain regularity conditions, slightly weaker than $\mathcal{D}_{1}-\mathcal{D}_{3}$ (see the next section and [4]), the score test

$$
\phi_{n}^{*}\left(X^{(n)}\right)= \begin{cases}1, & \text { if } \Delta_{n}\left(\vartheta_{0}\right)>c_{n} \\ 0, & \text { if } \Delta_{n}\left(\vartheta_{0}\right) \leq c_{n}\end{cases}
$$

based on

$$
\Delta_{n}\left(\vartheta_{0}\right)=\varphi_{n} \int_{\mathbb{A}_{n}} \frac{\dot{S}\left(\vartheta_{0}, x\right)}{S\left(\vartheta_{0}, x\right)} \pi^{(n)}(\mathrm{d} x)
$$

with

$$
c_{n}=z_{\alpha}-\frac{\gamma_{3, n}}{6}\left(1-z_{\alpha}^{2}\right), \quad \gamma_{3, n}=\varphi_{n}^{3} \int_{\mathbb{A}_{n}} \frac{\dot{S}\left(\vartheta_{0}, x\right)^{3}}{S\left(\vartheta_{0}, x\right)^{2}} \mathrm{~d} x
$$

is second order efficient, i.e., it satisfies

$$
\sup _{0 \leq u \leq K}\left|\beta_{n}\left(u, \phi_{n}^{*}\right)-\beta_{n}\left(u, \tilde{\phi}_{n}\right)\right|=O\left(\varepsilon_{n}^{2}\right),
$$

for any $K>0$. For proof see [4], Theorem 6. Indeed to establish the second order efficiency we obtain the following representation of distribution functions of $\Delta_{n}\left(\vartheta_{0}\right)$ and $\Lambda_{n}(u)$ under the local alternative $\vartheta_{u}$ :

$$
\begin{aligned}
& \beta_{n}\left(u, \phi_{n}^{*}\right)=\mathcal{N}\left(\frac{m_{n}(u)-c_{n}}{\eta_{n}}\right)-\frac{\gamma_{3, n}(u)}{6}\left(1-\left(u-z_{\alpha}\right)^{2}\right) n\left(u-z_{\alpha}\right)+O\left(\varepsilon_{n}^{2}\right) \\
& \beta_{n}\left(u, \tilde{\phi}_{n}\right)=\mathcal{N}\left(\frac{\mu_{n}(u)-b_{n}(u)}{\sigma_{n}(u)}\right)-\frac{\gamma_{3, n}^{\prime}(u)}{6}\left(1-\left(u-z_{\alpha}\right)^{2}\right) n\left(u-z_{\alpha}\right)+O\left(\varepsilon_{n}^{2}\right),
\end{aligned}
$$


where

$$
\begin{aligned}
m_{n}(u) & =\mathbf{E}_{\vartheta_{u}} \Delta_{n}\left(\vartheta_{0}\right)=\varphi_{n} \int_{\mathbb{A}_{n}} \frac{\dot{S}\left(\vartheta_{0}, x\right)}{S\left(\vartheta_{0}, x\right)}\left(S\left(\vartheta_{u}, x\right)-S\left(\vartheta_{0}, x\right)\right) \mathrm{d} x \\
\eta_{n}^{2} & =\mathbf{E}_{\vartheta_{u}}\left(\Delta_{n}\left(\vartheta_{0}\right)-m_{n}(u)\right)^{2}=\varphi_{n}^{2} \int_{\mathbb{A}_{n}} \frac{\dot{S}\left(\vartheta_{0}, x\right)^{2}}{S\left(\vartheta_{0}, x\right)^{2}} S\left(\vartheta_{u}, x\right) \mathrm{d} x \\
\mu_{n}(u) & =\mathbf{E}_{\vartheta_{u}} \Lambda_{n}(u)=\int_{\mathbb{A}_{n}}\left(\ln \frac{S\left(\vartheta_{u}, x\right)}{S\left(\vartheta_{0}, x\right)} S\left(\vartheta_{u}, x\right)-S\left(\vartheta_{u}, x\right)+S\left(\vartheta_{0}, x\right)\right) \mathrm{d} x, \\
\sigma_{n}^{2}(u) & =\mathbf{E}_{\vartheta_{u}}\left(\Lambda_{n}(u)-\mu_{n}(u)\right)^{2}=\int_{\mathbb{A}_{n}}\left(\ln \frac{S\left(\vartheta_{u}, x\right)}{S\left(\vartheta_{0}, x\right)}\right)^{2} S\left(\vartheta_{u}, x\right) \mathrm{d} x, \\
\gamma_{3, n}(u) & =\frac{\varphi_{n}^{3}}{\eta_{n}^{3}} \int_{\mathbb{A}_{n}} \frac{\dot{S}\left(\vartheta_{0}, x\right)^{3}}{S\left(\vartheta_{0}, x\right)^{3}} S\left(\vartheta_{u}, x\right) \mathrm{d} x \\
\gamma_{3, n}^{\prime}(u) & =\frac{1}{\sigma_{n}(u)^{3}} \int_{\mathbb{A}_{n}}\left(\ln \frac{S\left(\vartheta_{u}, x\right)}{S\left(\vartheta_{0}, x\right)}\right)^{3} S\left(\vartheta_{u}, x\right) \mathrm{d} x .
\end{aligned}
$$

Hence it suffices to show that

$$
\begin{aligned}
\sup _{0<u \leq K}\left|\gamma_{3, n}(u)-\gamma_{3, n}^{\prime}(u)\right| & =O\left(\varepsilon_{n}^{2}\right), \\
\sup _{0<u \leq K}\left|\frac{m_{n}(u)-c_{n}}{\eta_{n}}-\frac{\mu_{n}(u)-b_{n}(u)}{\sigma_{n}(u)}\right| & =O\left(\varepsilon_{n}^{2}\right) .
\end{aligned}
$$

The case $u=0$ corresponds to the size of $\tilde{\phi}_{n}$ which is equal to $\alpha$ and the size of $\phi_{n}^{*}$ given by $\alpha+O\left(\varepsilon_{n}^{2}\right)$. The latter follows from the Edgeworth expansion of distribution function of $\Delta_{n}\left(\vartheta_{0}\right)$ under the null hypothesis.

Representation of the power. Now we obtain the explicit representation of the power of $\phi_{n}^{*}$ (up to order $O\left(\varepsilon_{n}^{2}\right)$ ). The Taylor expansion

$$
S\left(\vartheta_{u}, x\right)=S\left(\vartheta_{0}, x\right)+\varphi_{n} u \dot{S}\left(\vartheta_{0}, x\right)+\frac{\varphi_{n}^{2} u^{2}}{2} \ddot{S}\left(\vartheta_{0}, x\right)+\frac{\varphi_{n}^{3} u^{3}}{3 !} S^{(3)}\left(\vartheta_{n}, x\right),
$$

for some intermediate point $\vartheta_{0}<\vartheta_{n}=\vartheta_{n}(u, x)<\vartheta_{u}$, implies that $m_{n}(u)-c_{n}=u-z_{\alpha}+\frac{\varphi_{n}^{3} u^{2}}{2} \int_{\mathbb{A}_{n}} \frac{\dot{S}\left(\vartheta_{0}, x\right) \ddot{S}\left(\vartheta_{0}, x\right)}{S\left(\vartheta_{0}, x\right)} \mathrm{d} x+\frac{\gamma_{3, n}}{6}\left(1-z_{\alpha}^{2}\right)+O\left(\varepsilon_{n}^{2}\right)$.

Similarly we obtain

$$
\eta_{n}^{2}=1+\varphi_{n}^{3} u \int_{\mathbb{A}_{n}} \frac{\dot{S}\left(\vartheta_{0}, x\right)^{3}}{S\left(\vartheta_{0}, x\right)^{2}} \mathrm{~d} x+O\left(\varepsilon_{n}^{2}\right)=1+u \gamma_{3, n}+O\left(\varepsilon_{n}^{2}\right) .
$$


Hence

$$
\begin{aligned}
\frac{m_{n}(u)-c_{n}}{\eta_{n}}=u-z_{\alpha} & +\frac{1-z_{\alpha}^{2}-3 u\left(u-z_{\alpha}\right)}{6} \gamma_{3, n}+ \\
& +\frac{\varphi_{n}^{3} u^{2}}{2} \int_{\mathbb{A}_{n}} \frac{\dot{S}\left(\vartheta_{0}, x\right) \ddot{S}\left(\vartheta_{0}, x\right)}{S\left(\vartheta_{0}, x\right)} \mathrm{d} x+O\left(\varepsilon_{n}^{2}\right) .
\end{aligned}
$$

On the other hand by the help of Taylor expansion we obtain

$$
\gamma_{3, n}(u)=\gamma_{3, n}+O\left(\varepsilon_{n}^{2}\right) .
$$

Therefore we obtain the following representation

$$
\beta_{n}\left(u, \phi_{n}^{*}\right)=\mathcal{N}\left(u-z_{\alpha}\right)+Q_{n}(u) n\left(u-z_{\alpha}\right)+O\left(\varepsilon_{n}^{2}\right),
$$

for any $u>0$, where the polynomial (in $u$ )

$$
Q_{n}(u)=\frac{u\left(z_{\alpha}-2 u\right)}{6} \gamma_{3, n}+\frac{\varphi_{n}^{3} u^{2}}{2} \int_{\mathbb{A}_{n}} \frac{\dot{S}\left(\vartheta_{0}, x\right) \ddot{S}\left(\vartheta_{0}, x\right)}{S\left(\vartheta_{0}, x\right)} \mathrm{d} x
$$

is of order $O\left(\varepsilon_{n}\right)$. The equation (4), refines the first order representation (1). Notice also that the the second order efficiency of $\phi_{n}^{*}$ implies that we have the same representation for the power of $\tilde{\phi}_{n}$.

\section{Power Loss}

The power function of a second order efficient test agrees with that of the most powerful test up to order $O\left(\varepsilon_{n}^{2}\right)$. Hence it is natural to consider the power loss of $\phi_{n}^{*}$, which is defined for any $u>0$ by

$$
r(u)=\lim _{n \rightarrow \infty} \varepsilon_{n}^{-2}\left(\beta_{n}\left(u, \tilde{\phi}_{n}\right)-\beta_{n}\left(u, \phi_{n}^{*}\right)\right) .
$$

Below $S^{(j)}(\vartheta, x)$ denotes the $j$ th derivative of $S(\vartheta, x)$ with respect to $\vartheta$. We write $\dot{S}(\vartheta, x)$ and $\ddot{S}(\vartheta, x)$ for the first and second derivatives, respectively. We consider the following conditions:

$\mathcal{D}_{1}$. The intensity function $S(\vartheta, x)$ is four times differentiable with respect to $\vartheta$ in a right neighborhood of $\vartheta_{0}$.

$\mathcal{D}_{2}$. The conditions $\mathcal{B}_{1}$ and $\mathcal{B}_{2}$ are satisfied for the stochastic integrals $\Delta_{n}\left(\vartheta_{0}\right)$ and $\Lambda_{n}(u)$ under $\mathcal{H}_{0}$ and $\mathcal{H}_{u}$ with some sequence $\varepsilon_{n} \rightarrow 0$, 
$\mathcal{D}_{3}$. There exists some functions $f_{j}(x), j=0, \ldots, 4, x \in \mathbb{A}_{n}$ not depending on $\vartheta$ such that $S(\vartheta, x) \geq f_{0}(x),\left|S^{(j)}(\vartheta, x)\right| \leq f_{j}(x), j=0, \ldots, 4$ for all $x \in \mathbb{A}_{n}$ and all $\vartheta$ in a right neighborhood of $\vartheta_{0}$. We suppose also that

$$
\begin{gathered}
\varphi_{n}^{k} \int_{\mathbb{A}_{n}} \frac{\left|f_{1}(x)\right|^{k}}{f_{0}(x)^{k-1}} \mathrm{~d} x=O\left(\varepsilon_{n}^{k-2}\right), \quad k=2,3,4 \\
\varphi_{n}^{2 j} \int_{\mathbb{A}_{n}} \frac{f_{j}(x)^{2}}{f_{0}(x)} \mathrm{d} x=O\left(\varepsilon_{n}^{2 j-2}\right), \quad j=2,3,4 .
\end{gathered}
$$

Example 1. Let $X^{(n)}$ be a realization of a Poisson process on the set $\mathbb{A}_{n}=[0, n]$ with positive intensity function $S(\vartheta, x)=\vartheta S(x)+\lambda$ (amplitude parameter) or $S(\vartheta, x)=S(\vartheta+x)+\lambda$ (phase parameter), where $S(\cdot)$ is a two times differentiable periodic function and $\lambda>0$ (dark current) is a known constant. In both cases the condition $\mathcal{D}_{3}$ is satisfied with $\varphi_{n} \sim C n^{-1 / 2}$ for some $C>0$ and $\varepsilon_{n}=n^{-1 / 2}$. For the frequency modulation model $S(\vartheta, x)=S(\vartheta x)+\lambda$ we have $\varphi_{n} \sim C n^{-3 / 2}$ and $\varepsilon_{n}=n^{-1 / 2}$.

Let $J_{n}$ denote the quantity:

$$
\begin{aligned}
J_{n} & =\varphi_{n}^{4} \int_{\mathbb{A}_{n}} \frac{\left(\dot{S}\left(\vartheta_{0}, x\right)^{2}-S\left(\vartheta_{0}, x\right) \ddot{S}\left(\vartheta_{0}, x\right)\right)^{2}}{S\left(\vartheta_{0}, x\right)^{3}} \mathrm{~d} x- \\
& -\left(\varphi_{n}^{3} \int_{\mathbb{A}_{n}} \frac{\dot{S}\left(\vartheta_{0}, x\right)\left(\dot{S}\left(\vartheta_{0}, x\right)^{2}-S\left(\vartheta_{0}, x\right) \ddot{S}\left(\vartheta_{0}, x\right)\right)}{S\left(\vartheta_{0}, x\right)^{3}} \mathrm{~d} x\right)^{2} .
\end{aligned}
$$

Note that $J_{n}=O\left(\varepsilon_{n}^{2}\right)$, by $\mathcal{D}_{3}$. We have the following theorem:

Theorem 2. Let the conditions $\mathcal{D}_{1}-\mathcal{D}_{3}$ be fulfilled. Then the power loss of $\phi_{n}^{*}$ with respect to the most powerful test $\tilde{\phi}_{n}$ is equal to

$$
r(u)=\frac{u^{3} n\left(u-z_{\alpha}\right)}{8} \lim _{n \rightarrow \infty}\left(\varepsilon_{n}^{-2} J_{n}\right)
$$

for any $u>0$.

Proof. By $\mathcal{D}_{2}$ we can write the following third order expansions:

$$
\begin{aligned}
\beta_{n}\left(u, \phi_{n}^{*}\right) & =\mathcal{N}\left(a_{n}\right)+\frac{\gamma_{3, n}(u)}{3 !} H_{2}\left(a_{n}\right) n\left(a_{n}\right)-\frac{\gamma_{4, n}(u)}{4 !} H_{3}\left(a_{n}\right) n\left(a_{n}\right)- \\
& -\frac{\gamma_{3, n}^{2}(u)}{72} H_{5}\left(a_{n}\right) n\left(a_{n}\right)+O\left(\varepsilon_{n}^{3}\right) \\
\beta_{n}\left(u, \tilde{\phi}_{n}\right) & =\mathcal{N}\left(A_{n}\right)+\frac{\gamma_{3, n}^{\prime}(u)}{3 !} H_{2}\left(A_{n}\right) n\left(A_{n}\right)-\frac{\gamma_{4, n}^{\prime}(u)}{4 !} H_{3}\left(A_{n}\right) n\left(A_{n}\right)- \\
& -\frac{\gamma_{3, n}^{\prime}(u)^{2}}{72} H_{5}\left(A_{n}\right) n\left(A_{n}\right)+O\left(\varepsilon_{n}^{3}\right),
\end{aligned}
$$


where

$$
\begin{aligned}
a_{n} & =\frac{m_{n}(u)-c_{n}}{\eta_{n}}, \quad A_{n}=\frac{\mu_{n}(u)-b_{n}(u)}{\sigma_{n}(u)} \\
\gamma_{r, n}(u) & =\frac{\varphi_{n}^{r}}{\eta_{n}^{r}} \int_{\mathbb{A}_{n}} \frac{\dot{S}\left(\vartheta_{0}, x\right)^{r}}{S\left(\vartheta_{0}, x\right)^{r}} S\left(\vartheta_{u}, x\right) \mathrm{d} x, \quad r=3,4 \\
\gamma_{r, n}^{\prime}(u) & =\frac{1}{\sigma_{n}(u)^{r}} \int_{\mathbb{A}_{n}}\left(\ln \frac{S\left(\vartheta_{u}, x\right)}{S\left(\vartheta_{0}, x\right)}\right)^{r} S\left(\vartheta_{u}, x\right) \mathrm{d} x, \quad r=3,4 .
\end{aligned}
$$

Using the Taylor expansions of $S\left(\vartheta_{u}, x\right)$ and $\ln \frac{S\left(\vartheta_{u}, x\right)}{S\left(\vartheta_{0}, x\right)}$, we get

$$
\begin{array}{ll}
\gamma_{4, n}(u)=\gamma_{4, n}+O\left(\varepsilon_{n}^{3}\right), & \gamma_{3, n}(u)^{2}=\gamma_{3, n}^{2}+O\left(\varepsilon_{n}^{3}\right) \\
\gamma_{4, n}^{\prime}(u)=\gamma_{4, n}+O\left(\varepsilon_{n}^{3}\right), & \gamma_{3, n}^{\prime}(u)^{2}=\gamma_{3, n}^{2}+O\left(\varepsilon_{n}^{3}\right) .
\end{array}
$$

Since $A_{n}-a_{n}=O\left(\varepsilon_{n}^{2}\right)$ (which follows from the second order efficiency of $\phi_{n}^{*}$ ), then

$$
\mathcal{N}\left(A_{n}\right)=\mathcal{N}\left(a_{n}\right)+\left(A_{n}-a_{n}\right) n\left(a_{n}\right)+O\left(\varepsilon_{n}^{4}\right) .
$$

Therefore

$$
\begin{aligned}
\beta_{n}\left(u, \phi_{n}^{*}\right) & =\mathcal{N}\left(a_{n}\right)+\frac{\gamma_{3, n}}{3 !} H_{2}\left(a_{n}\right) n\left(a_{n}\right)-\frac{\gamma_{4, n}}{4 !} H_{3}\left(a_{n}\right) n\left(a_{n}\right)- \\
& -\frac{\gamma_{3, n}^{2}}{72} H_{5}\left(a_{n}\right) n\left(a_{n}\right)+O\left(\varepsilon_{n}^{3}\right), \\
\beta_{n}\left(u, \tilde{\phi}_{n}\right) & =\mathcal{N}\left(a_{n}\right)+\left(A_{n}-a_{n}\right) n\left(a_{n}\right)+\frac{\gamma_{3, n}^{\prime}(u)}{3 !} H_{2}\left(a_{n}\right) n\left(a_{n}\right)- \\
& -\frac{\gamma_{4, n}}{4 !} H_{3}\left(a_{n}\right) n\left(a_{n}\right)-\frac{\gamma_{3, n}^{2}}{72} H_{5}\left(a_{n}\right) n\left(a_{n}\right)+O\left(\varepsilon_{n}^{3}\right) .
\end{aligned}
$$

Letting $\Delta=\Delta(u)=u-z_{\alpha}$ and taking into account the fact that $a_{n}-\Delta=$ $O\left(\varepsilon_{n}\right)$, we can write

$$
\begin{aligned}
\beta_{n}\left(u, \tilde{\phi}_{n}\right)-\beta_{n}\left(u, \phi_{n}^{*}\right) & =\left(A_{n}-a_{n}\right) n(\Delta)+ \\
& +\frac{H_{2}(\Delta) n(\Delta)}{6}\left(\gamma_{3, n}^{\prime}(u)-\gamma_{3, n}(u)\right)+O\left(\varepsilon_{n}^{3}\right) .
\end{aligned}
$$

Therefore we have to consider the terms $A_{n}-a_{n}$ and $\gamma_{3, n}^{\prime}(u)-\gamma_{3, n}(u)$. Since the investigation is at accuracy level $O\left(\varepsilon_{n}^{3}\right)$, we modify the threshold $c_{n}$ as follows:

$$
c_{n}=z_{\alpha}+\frac{\gamma_{3, n}}{6} H_{2}\left(z_{\alpha}\right)+\frac{\gamma_{4, n}}{4 !} H_{3}\left(z_{\alpha}\right)+\frac{\gamma_{3, n}^{2}}{72} H_{5}\left(z_{\alpha}\right) .
$$


With this constant the probability of error of the first kind of $\phi_{n}^{*}$ is equal to $\alpha+O\left(\varepsilon_{n}^{3}\right)$. Similarly the explicit form of $b_{n}(u)$, the threshold of $\tilde{\phi}_{n}$, can be written as:

$$
b_{n}(u)=\mu_{n}+\sigma_{n}\left(z_{\alpha}+\frac{\gamma_{3, n}^{\prime}}{6} H_{2}\left(z_{\alpha}\right)+\frac{\gamma_{4, n}^{\prime}}{4 !} H_{3}\left(z_{\alpha}\right)+\frac{\gamma_{3, n}^{\prime 2}}{72} H_{5}\left(z_{\alpha}\right)\right)+O\left(\varepsilon_{n}^{3}\right),
$$

where

$$
\begin{aligned}
\mu_{n} & =\mathbf{E}_{\vartheta_{0}} \Lambda_{n}(u)=\int_{\mathbb{A}_{n}}\left[\ln \frac{S\left(\vartheta_{u}, x\right)}{S\left(\vartheta_{0}, x\right)}-\frac{S\left(\vartheta_{u}, x\right)}{S\left(\vartheta_{0}, x\right)}+1\right] S\left(\vartheta_{0}, x\right) \mathrm{d} x, \\
\sigma_{n}^{2} & =\mathbf{E}_{\vartheta_{0}}\left(\Lambda_{n}(u)-\mu_{n}\right)^{2}=\int_{\mathbb{A}_{n}}\left(\ln \frac{S\left(\vartheta_{u}, x\right)}{S\left(\vartheta_{0}, x\right)}\right)^{2} S\left(\vartheta_{0}, x\right) \mathrm{d} x, \\
\gamma_{r, n}^{\prime} & =\frac{1}{\sigma_{n}^{r}} \int_{\mathbb{A}_{n}}\left(\ln \frac{S\left(\vartheta_{u}, x\right)}{S\left(\vartheta_{0}, x\right)}\right)^{r} S\left(\vartheta_{0}, x\right) \mathrm{d} x, \quad r=3,4 .
\end{aligned}
$$

Below for the sake of simplicity we use the following notations:

$$
I\left(r_{0}, r_{1}, r_{2}\right)=\int_{\mathbb{A}_{n}} \frac{\dot{S}\left(\vartheta_{0}, x\right)^{r_{1}} \ddot{S}\left(\vartheta_{0}, x\right)^{r_{2}}}{S\left(\vartheta_{0}, x\right)^{r_{0}}} \mathrm{~d} x
$$

for nonnegative integers $r_{0}, r_{1}, r_{2}$. The long but straightforward calculations show that (see Appendix)

$$
\begin{aligned}
A_{n}-a_{n} & =\frac{u^{3}}{8} \varphi_{n}^{4} I(1,0,2)-\frac{2 u^{3}-2 u^{2} z_{\alpha}-u\left(1-z_{\alpha}^{2}\right)}{4} \varphi_{n}^{4} I(2,2,1)+ \\
& +\frac{9 u^{3}-12 u^{2} z_{\alpha}-6 u\left(1-z_{\alpha}^{2}\right)}{24} \varphi_{n}^{4} I(3,4,0)+ \\
& +\frac{9 u^{3}-6 u^{2} z_{\alpha}+2 u\left(1-z_{\alpha}^{2}\right)}{24} \varphi_{n}^{6} I^{2}(2,3,0)+ \\
& +\frac{6 u^{3}-6 u^{2} z_{\alpha}-3 u\left(1-z_{\alpha}^{2}\right)}{12} \varphi_{n}^{6} I(2,3,0) I(1,1,1)- \\
& -\frac{u^{3}}{8} \varphi_{n}^{6} I(1,1,1)^{2}+O\left(\varepsilon_{n}^{3}\right) .
\end{aligned}
$$

Also for the term $\gamma_{3, n}^{\prime}(u)-\gamma_{3, n}(u)$ we find that

$$
\begin{aligned}
\gamma_{3, n}^{\prime}(u)-\gamma_{3, n}(u) & =\frac{3 u}{2} \varphi_{n}^{4} I(2,2,1)+\frac{3 u}{2} \varphi_{n}^{6} I(2,3,0)^{2}-\frac{3 u}{2} \varphi_{n}^{4} I(3,4,0)- \\
& -\frac{3 u}{2} \varphi_{n}^{6} I(2,3,0) I(1,1,1)+O\left(\varepsilon_{n}^{3}\right)
\end{aligned}
$$


See Appendix for some details. Combining these results yield

$$
\begin{aligned}
& n^{-1}(\Delta)\left(\beta_{n}\left(u, \tilde{\phi}_{n}\right)-\beta_{n}\left(u, \phi_{n}^{*}\right)\right)=\frac{u^{3}}{8} \varphi_{n}^{4} I(1,0,2)-\frac{u^{3}}{4} \varphi_{n}^{4} I(2,2,1)+ \\
& +\frac{u^{3}}{8} \varphi_{n}^{4} I(3,4,0)-\frac{u^{3}}{8} \varphi_{n}^{6} I(2,3,0)^{2}-\frac{u^{3}}{8} \varphi_{n}^{6} I(1,1,1)^{2}+ \\
& +\frac{u^{3}}{4} \varphi_{n}^{6} I(2,3,0) I(1,1,1)+O\left(\varepsilon_{n}^{3}\right)=\frac{u^{3}}{8} \varphi_{n}^{4}[I(1,0,2)-2 I(2,2,1)+ \\
& +I(3,4,0)]-\frac{u^{3}}{8} \varphi_{n}^{6}[I(2,3,0)-I(1,1,1)]^{2}+O\left(\varepsilon_{n}^{3}\right) .
\end{aligned}
$$

Hence for any $u>0$

$$
\beta_{n}\left(u, \tilde{\phi}_{n}\right)-\beta_{n}\left(u, \phi_{n}^{*}\right)=\frac{u^{3} n(\Delta)}{8} J_{n}+O\left(\varepsilon_{n}^{3}\right)
$$

where

$$
J_{n}=\varphi_{n}^{4}[I(1,0,2)-2 I(2,2,1)+I(3,4,0)]-\varphi_{n}^{6}[I(2,3,0)-I(1,1,1)]^{2} .
$$

This can be simplified as

$$
\begin{aligned}
J_{n} & =\varphi_{n}^{4} \int_{\mathbb{A}_{n}} \frac{\left(\dot{S}\left(\vartheta_{0}, x\right)^{2}-S\left(\vartheta_{0}, x\right) \ddot{S}\left(\vartheta_{0}, x\right)\right)^{2}}{S\left(\vartheta_{0}, x\right)^{3}} \mathrm{~d} x- \\
& -\left(\varphi_{n}^{3} \int_{\mathbb{A}_{n}} \frac{\dot{S}\left(\vartheta_{0}, x\right)\left(\dot{S}\left(\vartheta_{0}, x\right)^{2}-S\left(\vartheta_{0}, x\right) \ddot{S}\left(\vartheta_{0}, x\right)\right)}{S\left(\vartheta_{0}, x\right)^{3}} \mathrm{~d} x\right)^{2} .
\end{aligned}
$$

Note that by the Cauchy-Schwartz inequality;

$$
\begin{aligned}
& \left(\varphi_{n}^{3} \int_{\mathbb{A}_{n}} \frac{\dot{S}\left(\vartheta_{0}, x\right)\left(\dot{S}\left(\vartheta_{0}, x\right)^{2}-S\left(\vartheta_{0}, x\right) \ddot{S}\left(\vartheta_{0}, x\right)\right)}{S\left(\vartheta_{0}, x\right)^{3}} \mathrm{~d} x\right)^{2} \leq \\
\leq & \left(\varphi_{n}^{2} \int_{\mathbb{A}_{n}} \frac{\dot{S}\left(\vartheta_{0}, x\right)^{2}}{S\left(\vartheta_{0}, x\right)} \mathrm{d} x\right)\left(\varphi_{n}^{4} \int_{\mathbb{A}_{n}} \frac{\left(\dot{S}\left(\vartheta_{0}, x\right)^{2}-S\left(\vartheta_{0}, x\right) \ddot{S}\left(\vartheta_{0}, x\right)\right)^{2}}{S\left(\vartheta_{0}, x\right)^{3}} \mathrm{~d} x\right)= \\
= & \varphi_{n}^{4} \int_{\mathbb{A}_{n}} \frac{\left(\dot{S}\left(\vartheta_{0}, x\right)^{2}-S\left(\vartheta_{0}, x\right) \ddot{S}\left(\vartheta_{0}, x\right)\right)^{2}}{S\left(\vartheta_{0}, x\right)^{3}} \mathrm{~d} x .
\end{aligned}
$$

Hence as one expect $J_{n} \geq 0$, because the test $\tilde{\phi}_{n}$ has the maximum of power at the local alternative $\vartheta_{u}$. Furthermore $\mathcal{D}_{3}$ implies that the quantity $J_{n}$ is 
of order $O\left(\varepsilon_{n}^{2}\right)$. Therefore

$$
\varepsilon_{n}^{-2}\left(\beta_{n}\left(u, \tilde{\phi}_{n}\right)-\beta_{n}\left(u, \phi_{n}^{*}\right)\right)=\frac{u^{3} n(\Delta)}{8} \varepsilon_{n}^{-2} J_{n}+O\left(\varepsilon_{n}\right)
$$

which completes the proof of the theorem.

Example 2. (Amplitude Parameter.) Suppose that we observe a realization $X^{(n)}$ of a Poisson process on the set $\mathbb{A}_{n}=[0, n], n=1,2, \cdots$ with the intensity function

$$
S(\vartheta, x)=\vartheta S(x)+\lambda, \quad \vartheta>0
$$

where $\lambda$ is a known positive constant (dark-current) and $S(x)$ is a known, nonconstant, differentiable (with respect to $x$ ) and periodic function with period $\tau>0$. We have two hypotheses $\mathcal{H}_{0}: \vartheta=\vartheta_{0}$ against $\mathcal{H}_{1}: \vartheta>\vartheta_{0}$, where $\vartheta_{0}>0$. The intensity function $S(\vartheta, x)$ is supposed to be positive in a right neighborhood of $\vartheta_{0}$ and all $x$. We obtain the power loss of the test

$$
\phi_{n}^{*}\left(X^{(n)}\right)=\chi_{\left\{\Delta_{n}\left(\vartheta_{0}\right)>c_{n}\right\}}
$$

with

$$
c_{n}=z_{\alpha}+\frac{\gamma_{3, n}}{6} H_{2}\left(z_{\alpha}\right)+\frac{\gamma_{4, n}}{24} H_{3}\left(z_{\alpha}\right)+\frac{\gamma_{3, n}^{2}}{72} H_{5}\left(z_{\alpha}\right)
$$

where

$$
\begin{aligned}
\gamma_{3, n} & =\frac{1}{\tau A^{3} \sqrt{n}} \int_{0}^{\tau} \frac{S(x)^{3}}{\left(\vartheta_{0} S(x)+\lambda\right)^{2}} \mathrm{~d} x \\
\gamma_{4, n} & =\frac{1}{\tau A^{4} n} \int_{0}^{\tau} \frac{S(x)^{4}}{\left(\vartheta_{0} S(x)+\lambda\right)^{3}} \mathrm{~d} x \\
A^{2} & =\frac{1}{\tau} \int_{0}^{\tau} \frac{S(x)^{2}}{\vartheta_{0} S(x)+\lambda} \mathrm{d} x .
\end{aligned}
$$

The conditions $\mathcal{D}_{1}-\mathcal{D}_{3}$ are satisfied (see [4], pp. 202-204) with $\varepsilon_{n}=n^{-1 / 2}$ and $\varphi_{n}=n^{-1 / 2} A^{-1}\left(1+O\left(n^{-1}\right)\right) \rightarrow 0$. One can obtain

$$
\lim _{n \rightarrow \infty}\left(\varepsilon_{n}^{-2} J_{n}\right)=\tau\left(\frac{\int_{0}^{\tau} \frac{S(x)^{4}}{\left(\vartheta_{0} S(x)+\lambda\right)^{3}} \mathrm{~d} x}{\left(\int_{0}^{\tau} \frac{S(x)^{2}}{\vartheta_{0} S(x)+\lambda} \mathrm{d} x\right)^{2}}-\frac{\left(\int_{0}^{\tau} \frac{S(x)^{3}}{\left.\vartheta_{0} S(x)+\lambda\right)^{3}} \mathrm{~d} x\right)^{2}}{\left(\int_{0}^{\tau} \frac{S(x)^{2}}{\vartheta_{0} S(x)+\lambda} \mathrm{d} x\right)^{3}}\right) \equiv B .
$$

Hence the power loss is equal to

$$
r(u)=\frac{u^{3} n\left(u-z_{\alpha}\right)}{8} B .
$$


Example 3. (Frequency parameter) In this example we consider a strongly nonhomogeneous case with nonclassical rate $n^{-3 / 2}$ (instead of $n^{-1 / 2}$ in the i.i.d. case). Suppose that we observe a realization $X^{(n)}$ of a Poisson process on the set $\mathbb{A}_{n}=[0, n], n=1,2, \cdots$ with periodic intensity function

$$
S(\vartheta, x)=e^{\sin (\vartheta x)}, \quad \vartheta>0 .
$$

We have the simple hypothesis $\mathcal{H}_{0}: \vartheta=\vartheta_{0}$ against $\mathcal{H}_{1}: \vartheta>\vartheta_{0}$. We consider the test $\phi_{n}^{*}\left(X^{(n)}\right)=\chi_{\left\{\Delta_{n}\left(\vartheta_{0}\right)>c_{n}\right\}}$ based on the statistic

$$
\Delta_{n}\left(\vartheta_{0}\right)=\varphi_{n} \int_{0}^{n} x \cos \left(\vartheta_{0} x\right)\left(X^{(n)}(\mathrm{d} x)-e^{\sin \left(\vartheta_{0} x\right)} \mathrm{d} x\right)
$$

and the threshold

$$
c_{n}=z_{\alpha}+\frac{\gamma_{3, n}}{6} H_{2}\left(z_{\alpha}\right)+\frac{\gamma_{4, n}}{24} H_{3}\left(z_{\alpha}\right)+\frac{\gamma_{3, n}^{2}}{72} H_{5}\left(z_{\alpha}\right),
$$

where letting $\tau=\frac{2 \pi}{\vartheta_{0}}$ we have

$$
\begin{aligned}
\gamma_{3, n} & =\frac{C^{3}}{4 \tau \sqrt{n}} \int_{0}^{\tau} \cos ^{3}\left(\vartheta_{0} x\right) e^{\sin \left(\vartheta_{0} x\right)} \mathrm{d} x \\
\gamma_{4, n} & =\frac{C^{4}}{5 \tau n} \int_{0}^{\tau} \cos ^{4}\left(\vartheta_{0} x\right) e^{\sin \left(\vartheta_{0} x\right)} \mathrm{d} x \\
C^{-2} & =\frac{1}{3 \tau} \int_{0}^{\tau} \cos ^{2}\left(\vartheta_{0} x\right) e^{\sin \left(\vartheta_{0} x\right)} \mathrm{d} x .
\end{aligned}
$$

The conditions $\mathcal{D}_{1}-\mathcal{D}_{3}$ are satisfied (see [4], pp. 205-207) with $\varepsilon_{n}=n^{-1 / 2}$ and $\varphi_{n} \sim C n^{-3 / 2}$.

The power loss is equal to

$$
r(u)=\frac{9 \tau u^{3} n\left(u-z_{\alpha}\right)}{40} \frac{\int_{0}^{\tau} \sin ^{2}\left(\vartheta_{0} x\right) e^{\sin \left(\vartheta_{0} x\right)} \mathrm{d} x}{\left(\int_{0}^{\tau} \cos ^{2}\left(\vartheta_{0} x\right) e^{\sin \left(\vartheta_{0} x\right)} \mathrm{d} x\right)^{2}},
$$

for any $u>0$.

Representation of the power. We consider the explicit representation of the power of $\phi_{n}^{*}$ up to $O\left(\varepsilon_{n}^{3}\right)$. As we saw

$$
\begin{aligned}
\beta_{n}\left(u, \phi_{n}^{*}\right) & =\mathcal{N}\left(a_{n}\right)+\frac{\gamma_{3, n}}{3 !} H_{2}\left(a_{n}\right) n\left(a_{n}\right)-\frac{\gamma_{4, n}}{4 !} H_{3}\left(a_{n}\right) n\left(a_{n}\right)- \\
& -\frac{\gamma_{3, n}^{2}}{72} H_{5}\left(a_{n}\right) n\left(a_{n}\right)+O\left(\varepsilon_{n}^{3}\right) .
\end{aligned}
$$


Since $a_{n}-\Delta=O\left(\varepsilon_{n}\right)$, it can be written as

$$
\begin{aligned}
\beta_{n}\left(u, \phi_{n}^{*}\right) & =\mathcal{N}\left(a_{n}\right)+\frac{\gamma_{3, n}}{3 !} H_{2}\left(a_{n}\right) n\left(a_{n}\right)-\frac{\gamma_{4, n}}{4 !} H_{3}(\Delta) n(\Delta)- \\
& -\frac{\gamma_{3, n}^{2}}{72} H_{5}(\Delta) n(\Delta)+O\left(\varepsilon_{n}^{3}\right) .
\end{aligned}
$$

Remind that $\Delta=u-z_{\alpha}$. Indeed we have (see Appendix)

$$
\begin{aligned}
a_{n}-\Delta & =\frac{u^{2}}{2} \varphi_{n}^{3} I(1,1,1)+\frac{u^{3}}{6} \varphi_{n}^{4} I(1,1,0,1)+ \\
& +\frac{1-z_{\alpha}^{2}-3 u \Delta}{6} \varphi_{n}^{3} I(2,3,0)+ \\
& +\frac{9 u^{2} \Delta-2 u\left(1-z_{\alpha}^{2}\right)}{24} \varphi_{n}^{6} I(2,3,0)^{2}-\frac{u^{2} \Delta}{4} \varphi_{n}^{4} I(2,2,1)- \\
& -\frac{u^{3}}{4} \varphi_{n}^{6} I(2,3,0) I(1,1,1)+O\left(\varepsilon_{n}^{3}\right) .
\end{aligned}
$$

Taylor expansion yields

$$
\mathcal{N}\left(a_{n}\right)=\mathcal{N}(\Delta)+\left(a_{n}-\Delta\right) n(\Delta)-\frac{\left(a_{n}-\Delta\right)^{2} \Delta n(\Delta)}{2}+O\left(\varepsilon_{n}^{3}\right)
$$

and

$$
\begin{aligned}
\gamma_{3, n} H_{2}\left(a_{n}\right) n\left(a_{n}\right) & =\gamma_{3, n} H_{2}(\Delta) n(\Delta)+ \\
& +\Delta\left(2-H_{2}(\Delta)\right) n(\Delta) \gamma_{3, n}\left(a_{n}-\Delta\right)+O\left(\varepsilon_{n}^{3}\right)
\end{aligned}
$$

Note that

$$
\left(a_{n}-\Delta\right)^{2}=\left(\frac{u^{2}}{2} \varphi_{n}^{3} I(1,1,1)+\frac{1-z_{\alpha}^{2}-3 u \Delta}{6} \varphi_{n}^{3} I(2,3,0)\right)^{2}+O\left(\varepsilon_{n}^{3}\right)
$$

and

$\gamma_{3, n}\left(a_{n}-\Delta\right)=\frac{u^{2}}{2} \varphi_{n}^{6} I(2,3,0) I(1,1,1)+\frac{1-z_{\alpha}^{2}-3 u \Delta}{6} \varphi_{n}^{6} I(2,3,0)^{2}+O\left(\varepsilon_{n}^{3}\right)$.

Therefore we arrive at the following form

$$
\beta_{n}\left(u, \phi_{n}^{*}\right)=\mathcal{N}(\Delta)+n(\Delta)\left(r_{1}(n)+r_{2}(n)\right)+O\left(\varepsilon_{n}^{3}\right),
$$


where the terms $r_{i}(n)=O\left(\varepsilon_{n}^{i}\right), i=1,2$,

$$
\begin{aligned}
& r_{1}(n)=\frac{u^{2}}{2} \varphi_{n}^{3} I(1,1,1)+\frac{u z_{\alpha}-2 u^{2}}{6} \gamma_{3, n} \\
& r_{2}(n)=\frac{u^{3}}{6} \varphi_{n}^{4} I(1,1,0,1)+\frac{9 u^{2} \Delta-2 u\left(1-z_{\alpha}^{2}\right)}{24} \gamma_{3, n}^{2}- \\
& -\frac{u^{2} \Delta}{4} \varphi_{n}^{4} I(2,2,1)-\frac{u^{3}}{4} \varphi_{n}^{3} \gamma_{3, n} I(1,1,1)- \\
& -\frac{\Delta}{2}\left(\frac{u^{2}}{2} \varphi_{n}^{3} I(1,1,1)+\frac{1-z_{\alpha}^{2}-3 u \Delta}{6} \gamma_{3, n}\right)^{2}+ \\
& +\frac{\Delta\left(2-H_{2}(\Delta)\right)}{6}\left(\frac{u^{2}}{2} \varphi_{n}^{3} \gamma_{3, n} I(1,1,1)+\frac{1-z_{\alpha}^{2}-3 u \Delta}{6} \gamma_{3, n}^{2}\right)- \\
& -\frac{\gamma_{4, n}}{4 !} H_{3}(\Delta) n(\Delta)-\frac{\gamma_{3, n}^{2}}{72} H_{5}(\Delta) n(\Delta) .
\end{aligned}
$$

For the notations $I$ above, see (7) in the Appendix. Especially

$$
\varphi_{n}^{3} I(2,3,0)=\varphi_{n}^{3} \int_{\mathbb{A}_{n}} \frac{\dot{S}\left(\vartheta_{0}, x\right)^{3}}{S\left(\vartheta_{0}, x\right)^{2}} \mathrm{~d} x=\gamma_{3, n} .
$$

Note also that $r_{1}(n)=Q_{n}(u)$ given in the equation (4).

\section{Appendix}

In order to obtain (5) and ([6) we use the expansion of $S\left(\vartheta_{u}, x\right)$ about $\vartheta_{0}$ and the following Taylor expansions which precise the terms up to order $O\left(\varepsilon_{n}^{3}\right)$. Letting

$$
t=\frac{S\left(\vartheta_{u}, x\right)-S\left(\vartheta_{0}, x\right)}{S\left(\vartheta_{0}, x\right)}
$$

we have

$$
\begin{aligned}
& \ln (1+t)=t-\frac{1}{2} t^{2}+\frac{1}{3} t^{3}-\frac{1}{4} t^{4}+O\left(t^{5}\right) \\
& \ln ^{2}(1+t)=t^{2}-t^{3}+\frac{11}{12} t^{4}+O\left(t^{5}\right) \\
& \ln ^{3}(1+t)=t^{3}-\frac{3}{2} t^{4}+O\left(t^{5}\right) \\
& \ln ^{4}(1+t)=t^{4}+O\left(t^{5}\right) .
\end{aligned}
$$

Note that the conditions $\mathcal{D}_{3}$ imply that for the terms of order $O\left(t^{5}\right)$ we have

$$
\int_{\mathbb{A}_{n}} O\left(t^{5}\right) \mathrm{d} x=O\left(\varepsilon_{n}^{3}\right) .
$$


We use also the binomial expansions

$$
\begin{gathered}
(1+s)^{\frac{1}{2}}=1+\frac{1}{2} s-\frac{1}{8} s^{2}+O\left(s^{3}\right) \\
(1+s)^{-\frac{1}{2}}=1-\frac{1}{2} s+\frac{3}{8} s^{2}+O\left(s^{3}\right) \\
(1+s)^{-\frac{3}{2}}=1-\frac{3}{2} s+\frac{15}{8} s^{2}+O\left(s^{3}\right),
\end{gathered}
$$

for different values of $s=s_{n}(u)=O\left(\varepsilon_{n}\right)$ related to $\sigma_{n}^{2}(u), \sigma_{n}^{2}$ and $\eta_{n}^{2}$. We introduce the following notations:

$$
I\left(r_{0}, r_{1}, r_{2}, r_{3}\right)=\int_{\mathbb{A}_{n}} \frac{\dot{S}\left(\vartheta_{0}, x\right)^{r_{1}} \ddot{S}\left(\vartheta_{0}, x\right)^{r_{2}} S^{(3)}\left(\vartheta_{0}, x\right)^{r_{2}}}{S\left(\vartheta_{0}, x\right)^{r_{0}}} \mathrm{~d} x
$$

for nonnegative integers $r_{0}, r_{1}, r_{2}$ and $r_{3} \in\{0,1\}$. When $r_{3}=0$ we write $I\left(r_{0}, r_{1}, r_{2}\right)$ instead of $I\left(r_{0}, r_{1}, r_{2}, 0\right)$.

Remind that

$$
A_{n}=\frac{\mu_{n}(u)-b_{n}(u)}{\sigma_{n}(u)} \quad a_{n}=\frac{m_{n}(u)-c_{n}}{\eta_{n}} .
$$

These give:

$$
\begin{aligned}
\mu_{n}(u) & =\frac{u^{2}}{2}+\frac{u^{4}}{8} \varphi_{n}^{4} I(1,0,2)+\frac{u^{3}}{2} \varphi_{n}^{3} I(1,1,1)+\frac{u^{4}}{6} \varphi_{n}^{4} I(1,1,0,1)- \\
& -\frac{u^{3}}{6} \varphi_{n}^{3} I(2,3,0)-\frac{u^{4}}{4} \varphi_{n}^{4} I(2,2,1)+\frac{u^{4}}{12} \varphi_{n}^{4} I(3,4,0)+O\left(\varepsilon_{n}^{3}\right) \\
\sigma_{n}(u)^{-1} & =u^{-1}-\frac{u}{8} \varphi_{n}^{4} I(1,0,2)-\frac{1}{2} \varphi_{n}^{3} I(1,1,1)-\frac{u}{6} \varphi_{n}^{4} I(1,1,0,1)+ \\
& +\frac{u}{24} \varphi_{n}^{4} I(3,4,0)+\frac{3 u}{8} \varphi_{n}^{6} I(1,1,1)^{2}+O\left(\varepsilon_{n}^{3}\right) \\
b_{n}(u) & =-\frac{u^{2}}{2}+u z_{\alpha}-\frac{u^{4}-u^{3} z_{\alpha}}{8} \varphi_{n}^{4} I(1,0,2)-\frac{u^{3}-u^{2} z_{\alpha}}{2} \varphi_{n}^{3} I(1,1,1)- \\
& -\frac{u^{4}-u^{3} z_{\alpha}}{6} \varphi_{n}^{4} I(1,1,0,1)+\frac{2 u^{3}-u\left(1-z_{\alpha}^{2}\right)-3 u^{2} z_{\alpha}}{6} \varphi_{n}^{3} I(2,3,0)+ \\
& +\frac{2 u^{4}-u^{2}\left(1-z_{\alpha}^{2}\right)-3 u^{3} z_{\alpha}}{4} \varphi_{n}^{4} I(2,2,1)- \\
& -\frac{6 u^{4}-6 u^{2}\left(1-z_{\alpha}^{2}\right)-11 u^{3} z_{\alpha}}{24} \varphi_{n}^{4} I(3,4,0)- \\
& -\frac{4 u^{2}\left(1-z_{\alpha}^{2}\right)+3 u^{3} z_{\alpha}}{24} \varphi_{n}^{6} I(2,3,0)^{2}+ \\
& +\frac{2 u^{2}\left(1-z_{\alpha}^{2}\right)+3 u^{3} z_{\alpha}}{12} \varphi_{n}^{6} I(2,3,0) I(1,1,1)-\frac{u^{3} z_{\alpha}}{8} \varphi_{n}^{6} I(1,1,1)^{2}+O\left(\varepsilon_{n}^{3}\right) .
\end{aligned}
$$


Hence we arrive at

$$
\begin{aligned}
A_{n} & =u-z_{\alpha}+\frac{u^{3}}{8} \varphi_{n}^{4} I(1,0,2)+\frac{u^{2}}{2} \varphi_{n}^{3} I(1,1,1)+\frac{u^{3}}{6} \varphi_{n}^{4} I(1,1,0,1)- \\
& -\frac{3 u^{2}-\left(1-z_{\alpha}^{2}\right)-3 u z_{\alpha}}{6} \varphi_{n}^{3} I(2,3,0)- \\
& -\frac{3 u^{3}-6 u\left(1-z_{\alpha}^{2}\right)-12 u^{2} z_{\alpha}}{24} \varphi_{n}^{4} I(3,4,0)+ \\
& +\frac{4 u\left(1-z_{\alpha}^{2}\right)+3 u^{2} z_{\alpha}}{24} \varphi_{n}^{6} I(2,3,0)^{2}+ \\
& +\frac{3 u^{3}-3 u\left(1-z_{\alpha}^{2}\right)-6 u^{2} z_{\alpha}}{12} \varphi_{n}^{6} I(2,3,0) I(1,1,1)- \\
& -\frac{u^{3} z_{\alpha}}{8} \varphi_{n}^{6} I(1,1,1)^{2}+O\left(\varepsilon_{n}^{3}\right) .
\end{aligned}
$$

For the term $a_{n}$ we have

$$
\begin{aligned}
m_{n}(u) & =u+\frac{u^{2}}{2} \varphi_{n}^{3} I(1,1,1)+\frac{u^{3}}{6} \varphi_{n}^{4} I(1,1,0,1)+O\left(\varepsilon_{n}^{3}\right), \\
\eta_{n}^{-1} & =1-\frac{u}{2} \varphi_{n}^{3} I(2,3,0)-\frac{u^{2}}{4} \varphi_{n}^{4} I(2,2,1)+\frac{3 u^{2}}{8} \varphi_{n}^{6} I(2,3,0)^{2}+O\left(\varepsilon_{n}^{3}\right) .
\end{aligned}
$$

Therefore we can write

$$
\begin{aligned}
a_{n} & =u-z_{\alpha}+\frac{u^{2}}{2} \varphi_{n}^{3} I(1,1,1)+\frac{u^{3}}{6} \varphi_{n}^{4} I(1,1,0,1)+ \\
& +\frac{1-z_{\alpha}^{2}-3 u\left(u-z_{\alpha}\right)}{6} \varphi_{n}^{3} I(2,3,0)+ \\
& +\frac{9 u^{2}\left(u-z_{\alpha}\right)-2 u\left(1-z_{\alpha}^{2}\right)}{24} \varphi_{n}^{6} I(2,3,0)^{2}-\frac{u^{2}\left(u-z_{\alpha}\right)}{4} \varphi_{n}^{4} I(2,2,1)- \\
& -\frac{u^{3}}{4} \varphi_{n}^{6} I(2,3,0) I(1,1,1)+O\left(\varepsilon_{n}^{3}\right) .
\end{aligned}
$$

Hence one can obtain $A_{n}-a_{n}$ as in (5). Using the necessary Taylor expansions we get

$$
\begin{aligned}
\gamma_{3, n}(u) & =\varphi_{n}^{3} I(2,3,0)+u \varphi_{n}^{4} I(3,4,0)-\frac{3 u}{2} \varphi_{n}^{6} I(2,3,0)^{2}+O\left(\varepsilon_{n}^{3}\right), \\
\gamma_{3, n}^{\prime}(u)= & \varphi_{n}^{3} I(2,3,0)-\frac{u}{2} \varphi_{n}^{4} I(3,4,0)+\frac{3 u}{2} \varphi_{n}^{4} I(2,2,1)- \\
& -\frac{3 u}{2} \varphi_{n}^{6} I(2,3,0) I(1,1,1)+O\left(\varepsilon_{n}^{3}\right),
\end{aligned}
$$

which prove (6) 


\section{References}

[1] Bening, V.E. Asymptotic Theory of Testing Statistical Hypotheses. VSP, Utrecht, 2000.

[2] Daley, D.J. and Vere-Jones, D. An introduction to the theory of point processes. Springer, New York, 1988.

[3] Fazli Kh. Tests d'hypothèses asymptotiquement optimaux pour les processus de Poisson non homogènes, Thèse de doctorat de l'Université du Maine, 2006.

[4] Fazli Kh. Second order efficient test for inhomogeneous Poisson Processes, Statistical Inference for Stochastic Processes, 10:181-208 (2007).

[5] Krickeberg, K. Processus ponctuels en statistique. In Lecture Notes Math., Ecole d'Eté de Probabilités de Saint-Flour - 1980, Springer, 1982, LNM 929, 205-313.

[6] Kutoyants, Yu.A. Statistical Inference for Spatial Poisson Processes. Springer, New York, 1998.

[7] Noether, G.E. On a theorem of Pitman. Ann. Math.Statist. 26 (1955), 64-68.

[8] Pfanzagl, J. Asypmtotic Expansions for General Staticstical Models, Springer, New york, 1985.

[9] Pitman, E.J.G. Lecture Notes on Nonparametric Statistical Inferences. Lectures given at the University of Carolina, Institue of Statistics, 1948.

[10] Reiss, R.-D. A Course on Point Processes. Springer, New York, 1993.

[11] Rippley, B.D. Statistical Inference for Spatial Processes. Cambridge University Press, Cambridge, 1988.

[12] Roussas, G.G. Contiguity of Probability Measure. Cambridge University Press, Cambridge, 1972.

[13] Snyder, D.R. and Miller, M.I. Random Point Processes in Time and Space. Springer, New York, 1991. 